\title{
Preliminary Results from Intensity-Based CT-SPECT Fusion in I-131 Anti-B1 Monoclonal-Antibody Therapy of Lymphoma
}

\author{
Kenneth F. Koral, Ph.D. ${ }^{1}$ \\ Shuhong Lin, M.s. ${ }^{1}$ \\ Jeffrey A. Fessler, Ph.D. ${ }^{2}$ \\ Mark S. Kaminski, M.D. ${ }^{1}$ \\ Richard L. Wahl, M.D. ${ }^{1}$ \\ ${ }^{1}$ Department of Internal Medicine, University of \\ Michigan, Ann Arbor, Michigan. \\ ${ }^{2}$ Department of Electrical Engineering and \\ Computer Science, University of Michigan, Ann \\ Arbor, Michigan.
}

Presented in part at the Sixth Conference on Radioimmunodetection and Radioimmunotherapy of Cancer, Princeton, New Jersey, October 10-12, 1996.

Supported by grants R01 CA38790 (K.F.K.) and R01 CA 56794 (M.S.K.) from the National Cancer Institute, National Institutes of Health.

The authors thank Charles R. Meyer of the Department of Radiology, University of Michigan, for making both fusion programs available as well as for assistance in their use.

The contents of the article are solely the responsibility of the authors and do not necessarily represent the official views of the National Cancer Institute.

Address for reprints: Kenneth F. Koral, Ph.D., Nuclear-Medicine Research Center, 3480 Kresge III, 204 Zina Pitcher Place, University of Michigan Medical Center, Ann Arbor MI 481050552.

Received August 12, 1997; accepted September 5, 1997.
BACKGROUND. In treatment of non-Hodgkin's lymphoma patients with predoseplus-I-131-labeled anti-B1 (anti-CD20) monoclonal antibody, an intratherapy single photon emission computed tomography (SPECT) image is an important part of research estimates of tumor dosimetry. For that imaging, a computed tomography (CT)-SPECT fusion is used both to obtain an attenuation map for the spacealternating generalized expectation maximization reconstruction and to provide CT-based volumes of interest (VoI) to determine activity in tumors and organs. Fusion based on external, skin-surface markers has been used but may not correctly superimpose internal structures.

METHODS. A new algorithm, developed and implemented in the Department of Radiology, University of Michigan, and based on the mutual information of grayscale values, was investigated. Results from four anti-B1 therapy patients are presented.

RESULTS. In one patient, the new intensity-based fusion provided total reconstructed counts for kidneys that were higher than those produced by markerbased fusion; therefore, the VoI was probably located more accurately. In a second patient, after an acquisition that did not include any skin markers, the new algorithm produced counts/pixel that were similar for four of five tumors consistent with what is expected from an ideal therapy combined with accurate count density estimates. The fifth tumor was quite small and will have its final activity estimate moved toward consistency with the others after a recovery coefficient multiplication. For four tumors in two patients, direct comparison of the two algorithms yielded count totals that were different by no more than $7.2 \%$.

CONCLUSIONS. The use of CT-SPECT fusion and subsequent transfer of tumor VoI originally drawn in high-resolution CT space offers potential advantages for quantifying tumor uptake of radioactivity. A new, mutual-information-based fusion algorithm is usable without skin markers. Results indicate that the new fusion algorithm gives equal tumor count values within $7.2 \%$ compared with fusion based on external markers. It increases estimates of kidney activity by an average of $6.4 \%$. Cancer 1997;80:2538-44. (C) 1997 American Cancer Society.

KEYWORDS: medical oncology, therapy, fusion, lymphoma, I-131, SPECT, reconstruction, attenuation correction, activity quantification.

The mathematics of the superimposition (fusion) of a rigid body and its shifted, scaled, and rotated counterpart are well known. ${ }^{1}$ One technique for accomplishing such a fusion requires the identification of homologous (matched) points in the two image sets. This technique was applied to computed tomography-single photon emission computed tomography (CT-SPECT) fusion in monoclonal anti- 
body imaging at least as early as $1989 .^{2,3}$ In a similar approach, skin marking in SPECT has been combined with surface estimates from CT. ${ }^{4}$ We have used homologous points from skin markers in both CT and SPECT to allow fusion so that we could obtain attenuation maps for SPECT reconstruction from the CT images and also transfer volumes of interest (VoI) drawn on the CT images into the SPECT image space. ${ }^{5}$ The mathematics of the method has been briefly described elsewhere. ${ }^{6}$ There have been indications of good accuracy with the imaging of rigid phantoms. The root mean square difference in marker position was $1.5 \mathrm{~mm}$ with a five-marker fusion and $2.3 \mathrm{~mm}$ with a seven-marker fusion. ${ }^{7}$ In patient fusions, however, there can be problems, because the human body (with its organs and tumors) is not a "rigid body." Moreover, there is also a question of whether the internal organs maintain the same location relative to the external markers between the two scans that are taken at different times and with different modalities.

Therefore, we have investigated fusing images with a new method that is based on the intensities in the total volume of both image sets. In mathematical terms, the method depends on maximizing the mutual information of the two data sets. The iterative method plots the number of occurrences for a two-dimensional histogram that has CT intensity on one axis and SPECT activity per voxel on the other. As long as clusters in this histogram are strongest with a particular registration, the method converges. It is not necessary that two structures that have the same CT intensity must also have the same values for SPECT activity per voxel. However, for success to be achieved, it is important that the same structures appear in both modalities. The algorithm has been developed and implemented in the Department of Radiology, University of Michigan, and is described in a separate article. ${ }^{8}$ For the data sets presented here, the fusion is of the rigidbody type for both algorithms. With the marker-based method, we chose to not require perfect correspondence between modalities for all markers. For the intensity-based algorithm, convergence may not occur with the greater freedom of allowing a warping. However, both algorithms do allow for a change in voxel shape from one modality to another. This change can account for a uniform compression transversely coupled with an expansion longitudinally. We assume that such a change is caused by a respiration difference captured with the fast scanning times of a helical scanner. We choose to allow such a voxel change in some of the processing of our patient data.

In this article, we apply the mutual-information algorithm to SPECT and CT image sets for four new patients with non-Hodgkin's lymphoma. These pa- tients are being treated with a combination of nonradioactive and radioactive $\left({ }^{131}\right.$ I-labeled) anti-B1 (antiCD20) monoclonal antibody. ${ }^{9,10}$ The protein dose is $685 \mathrm{mg}$ nonradioactive and $15 \mathrm{mg}$ radioactive. We are most interested in the SPECT counts within tumor VoI defined by outlines drawn on CT by a trained radiologist. These counts lead to dosimetry estimates for the tumors. ${ }^{5}$

In general, the rationale for our use of the intensity-based algorithm is the hypothesis that the overlap of similar relative intensities throughout the image volume does produce the best superimposition of the tumors between modalities. According to the hypothesis, the skin markers are more susceptible to body contortions than are the internal features. To attempt validation of the superiority of the new algorithm compared with the marker-based one, we look for more counts within the VoI of kidneys that have uptake. We assume that this implies more accurate location of the VoI. To simply justify use of the new algorithm, we seek to show that counts within tumor VoI are not greatly changed.

\section{PATIENTS AND METHODS Data}

CT data are acquired with a General Electric (Milwaukee, WI) Genesis nonhelical or helical scanner. Data are reconstructed into $512 \times 512$ transverse matrices with 1-cm thick slices having no gaps or overlaps. For all fusion transformations, the original CT matrix is subsampled to a $256 \times 256$ matrix to reduce memory requirements. SPECT data are acquired after the therapeutic administration of anti-B1 when the patient body burden has dropped below $30 \mathrm{mCi}$. A threeheaded camera, the Picker (Cleveland, OH) Prism 3000 $\mathrm{XP}$, is used. Scans are accomplished with a fixed and identical radius of rotation for each head and a $360^{\circ}$ rotation of the gantry over 20 minutes. Sixty projection images are acquired by each head during continuous rotation. Preliminary results presented here are from the projections accumulated by head 1 and are reconstructed without compensation for Compton scattering.

There are two protocols for data acquisition. In one protocol, crossed lines are drawn on the skin of the patient at five locations. Four of these locations are chosen to be anterior, posterior, and right and left lateral, usually not in the same transverse plane. The fifth location is displaced longitudinally at least $10 \mathrm{~cm}$ from one of the others. During the CT scan, highly xray-absorbent, $1.5-\mathrm{mm}$ diameter, lead markers (Beekley Spots; Beekley, Bristol, CT) are placed at the intersections of the crossed lines. Patients lay supine on the usual CT table with hands over the head in the 
TABLE 1

Details of Image Acquisition and Processing

\begin{tabular}{llllll}
\hline & & Data acquisition & & \multicolumn{2}{c}{ Basis for fusion, type of scale } \\
\cline { 5 - 6 } Patient no. & Region & protocol & no. & First fusion & Second fusion \\
\hline 1 & Abdomen & 1 & 1 & Marker, fixed & Marker, fixed \\
2 & & 2 & Marker, fixed & Intensity, fixed \\
3 & Abdomen & 2 & & Intensity, fixed & Intensity, fixed \\
4 & Pelvis & 1 & 1 & Marker, variable & Intensity, fixed \\
& Abdomen & 1 & 2 & Marker, variable & Marker, variable \\
& & & & Intensity, variable & Intensity, variable \\
\hline
\end{tabular}

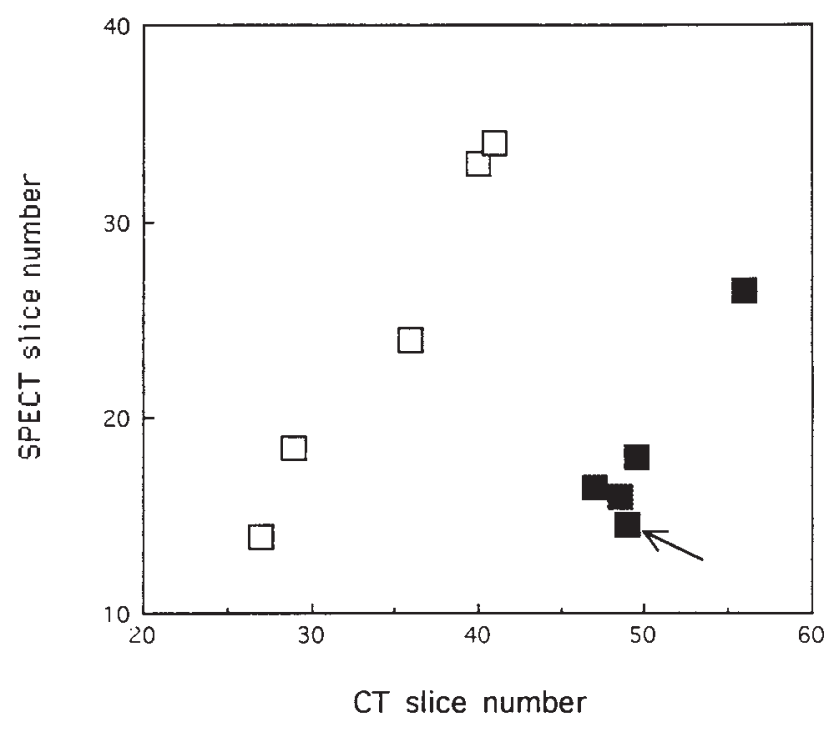

FIGURE 1. Plot of longitudinal marker location for two modalities. For Patient 1 (open squares), results are consistent with rigid-body assumption (i.e., the marker order from head to foot in the SPECT examination is the same as in the CT scan). For Patient 3 (black squares), results are inconsistent. Dropping the marker with the arrow improves the root mean square error for the marker-based fusion.

usual way. Normally, contrast is used. Care is taken to include the skin edges (to see the markers), and shallow breathing is allowed (to match the condition extant during the SPECT scan). Each marker is usually localized in a single transverse plane. During the SPECT scan, 3-mm diameter filter-paper circles saturated with $10-20 \mu \mathrm{Ci}$ of ${ }^{131} \mathrm{I}$ and sealed in plastic tape are similarly affixed to the skin. These markers are usually visualized in four to five of the 7.12-mm thick transverse planes. In the second protocol for data acquisition, markers are not used in either scan. No special provisions are made for either acquisition, except that the arms are placed over the head in a similar way for each.
The nuclear medicine data are reconstructed with filtered backprojection without attenuation correction using clinical software. The resultant image set provides the marker locations for marker-based fusion or image features (such as the skin edge or tip of the liver) so that control points can be chosen to produce an initial fusion for the mutual-information-based algorithm. Fusion parameters are obtained and stored by either (or both) of the two algorithms. These parameters are used to superimpose the CT data into the SPECT space, where it is extrapolated in energy to yield an attenuation map. ${ }^{5}$ The original SPECT projection data are then reconstructed with attenuation correction using the space-alternating generalized expectation maximization (SAGE) iterative algorithm ${ }^{11}$ plus the map. The stored fusion parameters are then reused to superimpose the attenuation-corrected SPECT into the CT space. Volumes of interest for the tumors (and perhaps kidneys) that have been outlined on the CT images are then carefully redrawn by hand on those images resident on a computer, which also contains the fused SPECT data. These VoI are stored. They are then recalled and applied to the SPECT reconstructions to obtain total counts within a tumor (or a kidney).

\section{Patients}

All four patients gave informed consent for the extra SPECT imaging. Patient 1 (University of Michigan, \#55) was a salvage therapy patient. He underwent abdominal scanning under Protocol 1. His image data were superimposed using both the older, marker-based algorithm and the newer mutual-information-based algorithm. In both cases, the attenuation map was obtained from the marker-based fusion. The second fusion was carried out once with each algorithm. For the intensity-based second fusion, three control points for the initial estimate were skin positions in the shape of an equilateral triangle in the corresponding slices that contained the inferior tip of the liver. A fourth 

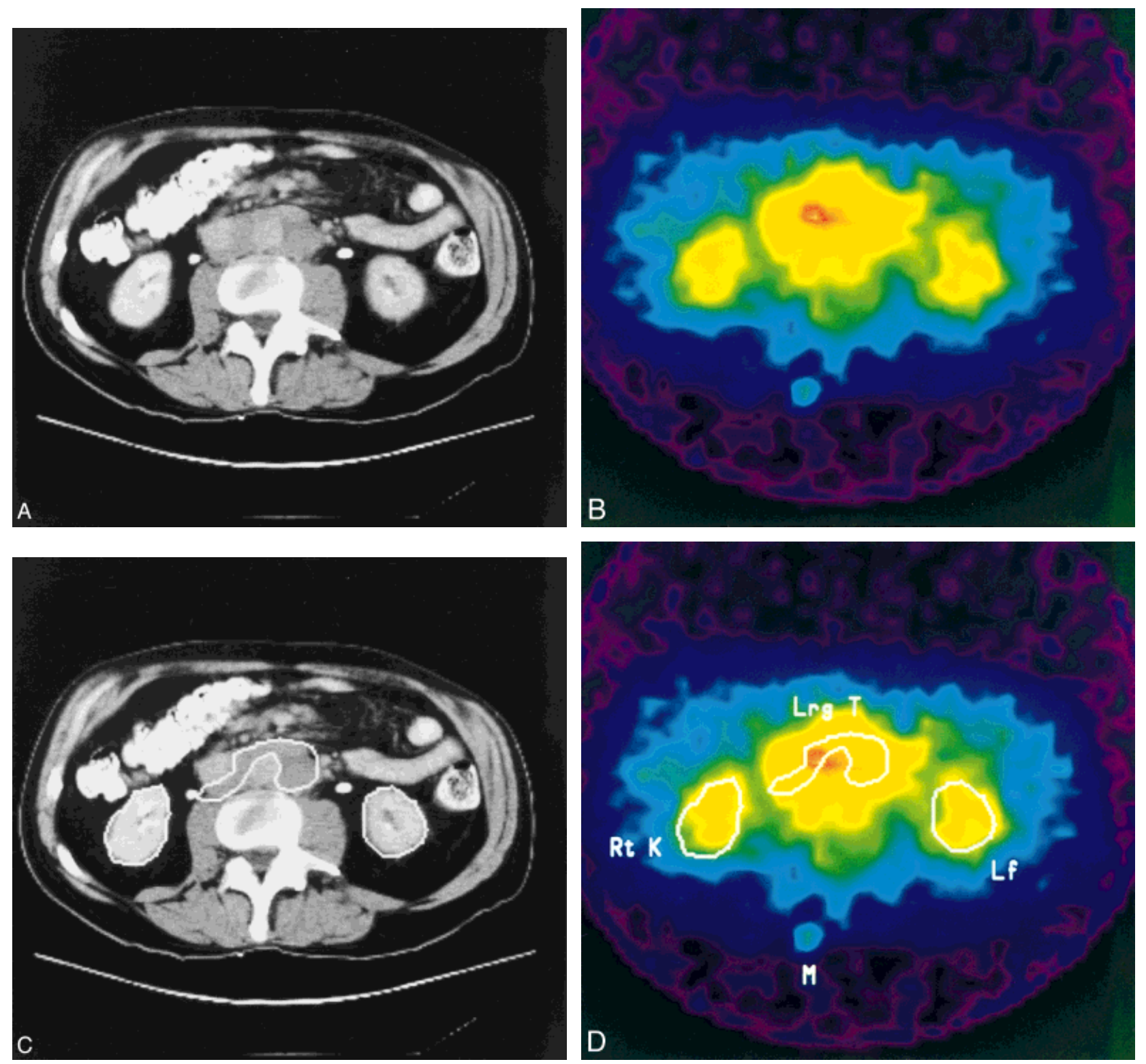

FIGURE 2. A slice for Patient 1. SPECT to CT fusion is with the mutual-information-based algorithm. X-ray CT without (A) and with (C) the handdrawn regions of interest are shown. The results from SPECT reconstruction in the same format are shown in B and D. Lrg T: tumor; Rt K: right kidney; Lf: left kidney; M: one of the skin-surface markers used in marker-based fusion.

was placed near the socket of the left hip. A fixed cubic voxel $1.33 \mathrm{~mm}$ on a side was specified for the $256 \times$ 256 CT scan and a similar voxel $7.12 \mathrm{~mm}$ on a side for the SPECT scan. The fusion took two iterations. Total elapsed CPU time was 3.5 minutes on a Digital Equipment Corporation (Nashua, NH) Model 3000/500× OSF/Alpha with a clock rate of $200 \mathrm{MHz}$ under the AVS programming environment.

Patient 2 (UF, \#1) was a patient in the up front treatment protocol in which the anti-B1 therapy was administered before any other therapy. He underwent abdominal scanning according to acquisition Protocol 2. The mutual-information-based fusion was used to go between spaces in both directions as is the usual case. Because no markers were imaged, there was no second final result to compare with that from the new algorithm.

Patient 3 (University of Michigan, \#64) was a second salvage therapy patient who underwent abdominal SPECT. Her attenuation map was obtained after a fusion based on the best four of five markers. A final mutual-information-based fusion appeared to be 


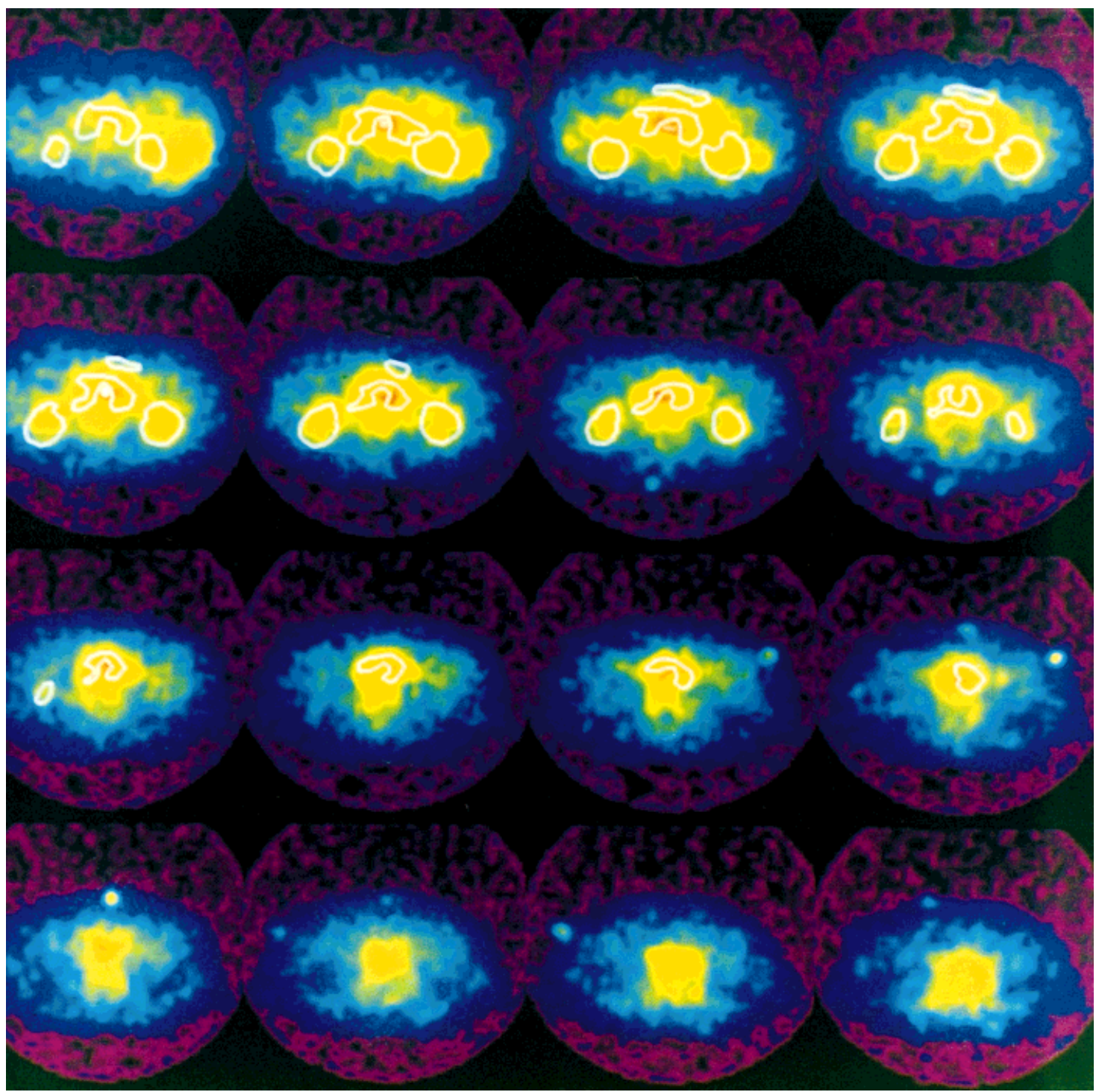

FIGURE 3. A series of 16 consecutive slices with regions of interest for Patient 1 . Progression is from head to feet. The slice shown in Figure 2 is the seventh in this series. A second, smaller, more anterior tumor appears in the third to sixth images. Three additional skin-surface markers are imaged most clearly in the 12th, 13th, and 15th slices.

quite successful. However, VoI could not be drawn on the CT images because excess fluid was not distinct from the tumor. Therefore, a final tumor evaluation of the patient is not included in this article.

Patient 4 (UF, \#5) was a second patient from the up front treatment protocol. She underwent abdominal scanning with markers using a GE helical scanner. In her case, we processed the raw projection data using each fusion method independently. For the mutual-information algorithm, a visually unsatisfactory CT-to-SPECT fusion was obtained with fixed-size cubic voxels. Improvement was obtained by permitting a SPECT voxel that was effectively noncubic, so we chose to use this fusion. Comparison of the results with those from marker-based fusion was performed. Table 1 summarizes both the imaging and processing for all four patients.

\section{RESULTS}

A problem that can occur with a marker-based algorithm can be seen by comparing results from two patients imaged under data acquisition Protocol 1. The SPECT slice number is plotted against the CT slice number for each marker for both patients in Figure 1. For Patient 1, the order from head to foot in the SPECT examination is the same as in the CT scan, whereas 
TABLE 2

Comparison of Two Fusion Methods for Patient 1

\begin{tabular}{llll}
\hline & \multicolumn{2}{c}{ Counts (arbitrary units) } & \\
\cline { 2 - 3 } & $\begin{array}{l}\text { Fusion using } \\
\text { markers }\end{array}$ & $\begin{array}{l}\text { Fusion using } \\
\text { intensity }\end{array}$ & $\begin{array}{l}\text { Difference } \\
(\%)\end{array}$ \\
\hline Leature & 10.8 & 11.1 & +3.2 \\
Right kidney & 8.24 & 9.04 & +9.6 \\
Large tumor & 17.0 & 17.6 & +4.0 \\
Small tumor & 1.50 & 1.39 & -7.2 \\
\hline
\end{tabular}

for Patient 3 it is not. The flexibility of the human body is thought to be the cause of the difference for Patient 3. (The patient tables are different, and no body mold or other restraining device is used.)

The traditional algorithm does find the solution to the fusion which has the least mean square distance between the input markers, even for Patient 3 , but this distance is large at $6.5 \mathrm{~mm}$. A possible solution is a warping algorithm, but this has not yet been attempted with our data. Another solution is to choose only a subset of the five markers. The use of a particular four markers yields a least square error of only 1.8 $\mathrm{mm}$ for Patient 3. It is our opinion that the discrepancy with all five markers $(6.5 \mathrm{~mm})$ is larger than is ascribable to experimental error in placing the markers over the ink marks or in establishing the locations of the markers in the image. There is such a large improvement (more than a factor of three) by dropping one marker that the four-marker fusion is kept. (Presumably the stretching and twisting of the body affected one marker disproportionately. This disproportionality is possible because the markers are located relatively far from each other.) Another solution for such patients that we also are investigating is the mutualinformation (intensity-based) algorithm.

For Patient 1, a slice from the final reconstruction after intensity-based fusion is shown in Figure 2. The CT image for the slice with and without region of interest (RoI) is shown in A and C. The reconstructed SPECT slice with and without the same RoI is shown in B and D. The outlined tumor $(\operatorname{Lrg} T$ in $D)$ is surrounded by activity from other structures and/or activity from the long tales of the ${ }^{131}$ I point-spread function. The cooler kidneys have activity apparently more bounded by their RoI.

Sixteen reconstructed slices for Patient 1 are shown in Figure 3. The slice of Figure 2 is the seventh in the series. A second smaller tumor appears in the third through sixth images of the series. It is clear that the drawn RoI would have been very difficult to realize without fusion plus transfer of VoI.
TABLE 3

Count Density in the Five Tumors of Patient 2

\begin{tabular}{lll}
\hline Tumor label & Volume (pixels) & $\begin{array}{l}\text { Count density } \\
\text { ("counts"/pixel, } \\
\text { arbitrary units) }\end{array}$ \\
\hline Superior small & 558 & 1.31 \\
Medium & 2,952 & 1.47 \\
Superior large & 18,115 & 1.60 \\
Inferior small & 159 & 0.41 \\
Inferior large & 11,104 & 1.67 \\
\hline
\end{tabular}

TABLE 4

Comparison of Two Fusion Methods for Patient 4

\begin{tabular}{llll}
\hline & \multicolumn{2}{c}{ Counts (arbitrary units) } & \\
\cline { 2 - 3 } & $\begin{array}{l}\text { Fusion using } \\
\text { markers }\end{array}$ & $\begin{array}{l}\text { Fusion using } \\
\text { intensity }\end{array}$ & $\begin{array}{l}\text { Difference } \\
(\%)\end{array}$ \\
\hline Large tumor & 71.7 & 68.2 & -4.8 \\
Small tumor & 0.29 & 0.28 & -2.3 \\
\hline
\end{tabular}

Locations for RoI in individual slices with the mutual-information-based fusion are visually similar to those with the marker-based fusion; a numeric comparison of the "reconstructed count" result is shown in Table 2. Sizes of the left and right kidneys in voxels are 7532 and 6595, respectively. Those of the large and small tumor are 10,191 and 1196. The left and right kidneys have $3.2 \%$ and $9.6 \%$ more counts, respectively, with the new superimposition. Our interpretation is that the VoI are more accurately placed over the true, higher activity location of those organs with the mutual-informationbased fusion. The "counts" for the large and small tumor change by +4.0 and $-7.2 \%$, respectively. In this case, the desirable direction of a count change is not known. We can only say that the new algorithm changes activity estimates by no more than $7.2 \%$ from those with the marker-based algorithm.

For Patient 2, there is little kidney uptake of the monoclonal antibody. However, there are five tumors identifiable from the CT scan. The "count" density in "counts"/pixel is itemized for these tumors in Table 3. Assuming that tumor uptake per gram should be similar for the different tumors, it is encouraging that the count density is less than $28 \%$ different for four of five tumors (range, 1.31-1.67) and 69\% lower only for the smallest. This smallest tumor will have its final value for activity per unit volume considerably increased relative to the others by multiplication by a large recovery coefficient. ${ }^{5}$

The final reconstructed images for Patient 4 with 
each fusion procedure were similar in appearance as for Patient 1 . The patient did not have kidney uptake distinct from that of the surrounding region. Therefore, a comparison of counts within kidney VoI would not be readily interpretable. A comparison of the counts within the tumor VoI is shown in Table 4. The large tumor occupied 46,012 pixels, whereas the small one only covered 207 . The results show slightly smaller differences between the fusion methods than in the case of Patient 1.

\section{DISCUSSION}

An advantage of the new algorithm is the possibility of accomplishing fusion even if (1) attenuating markers are not placed on the skin during the CT scan, (2) ink marks are not successfully maintained during the time between the CT scan and the therapy SPECT scan (2-3 weeks for our anti-B1 patients), or (3) the patient does not agree to the small amount of radiation exposure to the skin from placement of radioactive markers during the SPECT scan. A second advantage is potentially increased accuracy in superimposition of the CT tumor outline on the SPECT image.

In the present results, an indication of possible superiority of the intensity-based algorithm is that in a patient in whom there was identifiable kidney uptake, the new algorithm produced $3.2 \%$ (left) and 9.6\% (right) more activity than the marker-based algorithm, suggesting more proper placement of the VoI. Hints that use of the new algorithm is justified are twofold. First, in a patient with five tumors for which the new algorithm was used exclusively, the activity density was quite uniform in four of five tumors, in agreement with the expectation for the ideal biological case. Second, when direct comparison between the two algorithms was possible, in four tumors contained in two patients, the activity difference was never more than $7.2 \%$.

\section{REFERENCES}

1. Kessler ML, Pitluck S, Petti P, Castro JR. Integration of multimodality imaging data for radiotherapy treatment planning. Int J Radiat Oncol Biol Phys 1991;21:1653-67.

2. Kramer EL, Noz ME, Sanger JJ, Megibow AJ, Maguire GQ. CT-SPECT fusion to correlate radiolabeled monoclonal antibody uptake with abdominal CT findings. Radiology 1989; 172:861-5.

3. Kramer EL, Reddy D, Noz ME, Heelan R, Rusch V, Grahma $\mathrm{M}$, et al. "Fusion" of CT and radiolabeled antibody (AB) SPECT to improve mediastinal staging of patients with lung cancer [abstract]. J Nucl Med 1990;31:798.

4. Scott AM, Macapinlac H, Zhang J, Daghighian F, Montemayor N, Kalaigian H, et al. Image registration of SPECT and CT images using an external fiduciary band and three-dimensional surface fitting in metastatic thyroid cancer. J Nucl Med 1995;36:100-3.

5. Koral KF, Zasadny KR, Kessler ML, Luo J-q, Buchbinder SF, Kaminski MS, et al. CT-SPECT fusion plus conjugate views for determining dosimetry in I-131-MoAb therapy of lymphoma patients. J Nucl Med 1994;35:1714-20.

6. Wahl RL, Quint LE, Cieslak RD, Aisen AM, Koeppe RA, Meyer CR. "Anatometabolic" tumor imaging: fusion of FDG PET with CT or MRI to localize foci of increased activity. J Nucl Med 1993;34:1190-7.

7. Luo J. Dual window scatter correction in quantitative single photon emission computed tomography. Doctoral dissertation. Oakland University, 1993.

8. Meyer CR, Boes JL, Kim B, Bland PH, Zasadny KR, Kison PV, et al. Demonstration of accuracy and clinical versatility of mutual information for automatic multimodality image fusion using affine and thin-plate spline warped geometric deformations. Med Image Analysis 1997;1:195-206. Available at http://med714.bham.ac.uk/mia/Voll/No3/index.html

9. Kaminski MS, Zasadny KR, Francis IR, Milik AW, Ross CW, Moon SC, et al. Radioimmunotherapy of B-cell lymphoma with [131-I] anti-B1 (anti-CD20) antibody. $N$ Engl J Med 1993;329:459-65.

10. Kaminski MS, Zasadny KR, Francis IR, Fenner MC, Ross CW, Milik AW, et al. Iodine-131-anti-B1 radioimmunotherapy for B-cell lymphoma. J Clin Oncol 1996; 14:1974-81.

11. Fessler JA, Hero AO. Penalized maximum-likelihood image reconstruction using space-alternating generalized (EM) algorithms. IEEE Trans Image Processing 1995;4:1417-29. 